\title{
Applications of Nanomedicine in Antibacterial Medical Therapeutics and Diagnostics
}

\author{
Liam Matthews ${ }^{1}$, Rupinder K Kanwar ${ }^{1}$, Shufeng Zhou ${ }^{2}$, Vasu Punj ${ }^{3}$ and Jagat R. Kanwar ${ }^{*}, 1$ \\ ${ }^{I}$ BioDeakin, Institute for Technology \& Research Innovation, Deakin University, Geelong, Technology \\ Precinct, Pigdons Road, Waurn Ponds, Geelong, Victoria 3217. Australia \\ ${ }^{2}$ School of Health Sciences, RMIT University, Bundoora, Victoria-3083. Australia \\ ${ }^{3}$ Department of Medicine, University of Pittsburgh, Pittsburgh, Pennsylvania-15213
}

\begin{abstract}
The need for new and effective/efficient antibacterial therapeutics and diagnostics is necessary if we want to be able to maintain and improve the protection against pathogenic bacteria. Bacteria are becoming increasingly resistant to traditionally used antibiotics and as a result are a major health concern. The number of deaths and hospitalizations due to bacteria is increasing. Current methods of bacterial diagnostics are inefficient as they lack speed and ultra sensitivity and cannot be performed on site. This is where nanomedicine is playing a vital role. The discovery of new and innovative materials through the improvement in fabrication techniques has seen the establishment of an influx of novel antibacterial therapeutics and diagnostics. The goal of this review is to highlight the research that has been done through the implementation of nanomaterials and nanotechnologies for antibacterial medical therapeutic and diagnostic.
\end{abstract}

\section{INTRODUCTION}

Pathogenic bacteria remain a major health concern, which are responsible for causing a large number of deaths and hospitalizations each year. Although we have current treatments such as antibiotics, bacteria are gaining resistance to these therapeutics at an alarming rate. That is why new therapeutic and diagnostic treatments are necessary if we want to be prepared against known and unknown pathogenic bacterial infections. A range of potential solutions have been researched in hope that new treatments and diagnostic techniques will be developed. A large group of these studies includes the implementation of nanotechnologies and nanomaterials to create new antibacterial nanomedicines that increased effectiveness and efficiency [1,2]. Nanome-dicine is defined as the monitoring, repair, construction and control of human biological systems at the molecular level, using engineered nanodevices and nanostructures. This is the field of science and technology for diagnosing, treating and preventing disease and traumatic injury, of relieving pain, and of preserving and improving human health, using molecular tools and molecular knowledge of the human body. Nanotechnology is the area of science which deals with developing and producing extremely small tools and machines by controlling the arrangement of individual atoms thorough, inexpensive control of the structure of matter based on molecule-by-molecule control of products and byproducts; the products and processes of molecular manufacturing, including molecular machinery. This review looks at a range of new potential antibacterial therapeutics and diagnostics which implements nanotechnology, most of which are in early stages of research but has already shown

*Address correspondence to this author at the BioDeakin, Institute for Technology \& Research Innovation (ITRI), Geelong Technology Precinct (GTP), Deakin University, Pigdons Road, Waurn Ponds, Geelong, Victoria 3217, Australia; Tel: 0061-3 -5227 1148; Fax: 0061-3-5227 3402; E-mail: jagat.kanwar@deakin.edu.au positive signs of application into nanomedical therapeutics and diagnostics. With the use of new novel materials and technologies it seems that we will be able to create new antibacterial therapeutics that are more effective in combination with rapid and ultra sensitive bacterial diagnostics, which will hopefully allow us to obtain further protection against harmful bacteria. Nanocarriers have provided a novel platform for target-specific delivery of therapeutic agents. Over the past decade, several delivery vehicles have been designed based on different nanomaterials, such as polymers, dendrimers, liposomes, nanotubes, and nanorods. These nanoparticles have recently emerged as an attractive candidate for delivery as well as for diagnosis [1-6].

In this review we will write the comparative and systematic studies on nanotechnology based therapeutics and diagnostics on pathogenic bacteria which will give a complete picture of nanomedicine to researchers, patients and clinicians on treatments and diagnosis. This review will give new growing prospective for improving disease management and control in the future.

\section{THERAPEUTICS}

\section{Synthetic Antibacterial Nanomedicines}

\section{Carbon Nanotubes and Fullerenes}

Since the discovery of carbon nanotubes they have flourished and have been applied in a broad range of areas in science and in a way represent the momentum of nanotechnology itself. They have been sourced due to their unique optical, thermal, magnetic, high strength properties as well as the ability to be conductive, almost all of which can be tuned depending on the dimensions of the nanotube (Fig. 1). As of late they have been used in medical research in applications such as drug delivery and as components in 

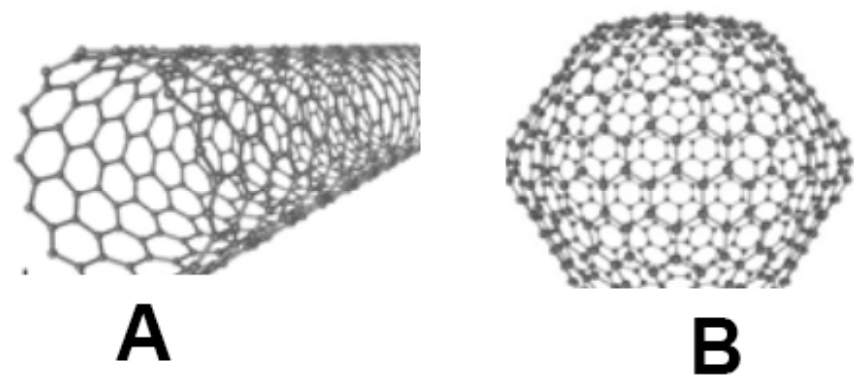

Fig. (1). The structures of (A) A single-walled nanotube (B) A fullerene $[1,2]$.

medical nanodevices. They have also been noted for their apparent toxicity to biological systems, as they themselves are so small that they act like fibers, and have the same toxicological impacts of other known toxic fibers, for example asbestos. A recent study was performed that looked into the antimicrobial properties of carbon nanotubes and showed that they are strong antibacterial agents [2]. The study conducted by Kang used low metal content, well characterized, narrowly distributed, pristine Single walled nanotubes (SWNTs), which are to observe bactericidal activity on the bacterium Escherichia coli K12. The bacteria were incubated with the SWNTs for 60-mins and microbial viability was checked and showed that over $80 \%$ of the microbes were killed. The suggested mode of death was the direct interaction of the SWNTs with the bacterial cell membranes causing significant membrane damage [7]. Similar research using nanotubes of different materials such as peptides and lipids also have shown the same mode of toxicity on microbes, suggesting that the nanotubes are able to physically penetrate the cell membranes to their physical dimensions leading to cell death [8]. Fullerene $\mathrm{C}_{60}$ is another carbon structure that has been investigated as an antimicrobial agent, as a means of identifying environmental impacts of fullerene $\mathrm{C}_{60}$. Research was conducted to test the toxicity of the fullerene molecules on two species of bacteria, E.coli DH5 $\alpha$ and Bacillus Subtilis, the former being a gram negative and the latter being gram positive [9]. The study showed that the fullerenes did act as bactericidal agents on both bacteria types, but it was also identified that differences in physical dimension of fullerene $\mathrm{C}_{60}$ types have alternative effects therefore more specific testing would be required [9].

\section{Silver Nanoparticles}

Silver has long been used in medicine, even in ancient times it was used as a preservative and also to reduce inflammation and prevent against infection of wounds [10]. It is due to this prior knowledge that further studies using silver as antibacterial agents has been perused, and bulk silver has already been used in appliances that are prone to microbial contamination, such as a fridge with an internal silver lining that was developed by LG (www.lge.com). With the development of more advanced materials and advanced fabrication techniques, nano sized materials can be easily produced, and differ from their macro counterparts, exhibiting new and enhanced properties. Silver is no exception, scaling down silver to the nanoscale provides a higher specific surface area and a higher fraction of surface atoms that improves its antimicrobial activity compared with bulk silver. A study was performed to test the effectiveness of silver nanoparticle antimicrobial activity using $E$.coli was undertaken by a Sonda [11]. In the study $10^{5} \mathrm{CFU}$ of gram negative $E$. coli colonies were grown on LB agar plates with different concentrations of Ag-nanoparticles. Ag-NPs at concentrations of $10 \mu \mathrm{g} \mathrm{cm}^{-3}$ showed inhibition of bacterial growth by up to $70 \%$, and at concentrations of 50-60 $\mu \mathrm{g} \mathrm{cm}$ 3 there was $100 \%$ inhibition of bacterial growth [12] A SEM and TEM analysis showed the accumulation of the nanoparticles within membranes and some were able to penetrate into the cell, the SEM also highlights the damage caused by the Ag-NPs where visible "pits" can be observed.

Silver ions antimicrobial activity is believed to work by impairing DNAs ability to replicate and through the inactivation of key proteins by denaturation when they are bound together [11]. Although it is still unknown what type of interaction takes place between the nanoparticles and the constituents of the outer membrane. Another study showed that with the use of silver nanoparticles in conjunction with antibiotics such as penicillin G, amoxicillin, erythromycin, clindamycin and vancomycin, provided an increase in the effectiveness of the antibiotics [12]. Silver nanoparticles show great potential as antimicrobial agents in applications, as they are of low cost and easily synthesized, and could be used to treat material surfaces to provide highly effective antibacterial materials, medical equipment such as in and devices and paints.

\section{Bioactive Glasses}

Bioactive glasses are a group of novel new materials that have been researched due to their interesting characteristics, and have already been used in clinical environments such as a disinfectant in dentistry. There is a range of different bioactive glasses but only one has been approved by FDA, known as $45 \mathrm{~S} 5$ and is composed of $\mathrm{SiO}_{2}-\mathrm{Na}_{2} \mathrm{O}-\mathrm{CaO}-\mathrm{P}_{2} \mathrm{O}_{5}$, and is currently used in dentistry [13]. It is well known that bioactive glasses in a solution exhibit a mild antimicrobial activity, due to the release of their ionic compounds and intake of $\mathrm{H}_{3} \mathrm{O}^{+}$protons it is able alter the local environment by increasing alkalinity of the solution. This in turn disrupts bacteria that are tolerant to the high-pH change. A study was conducted by Waltimo [13] to test if the hypothesis that nanometric bioactive glass should have a greater antimicrobial activity than when compared with regular bioactive glasses due to a higher specific surface area, which in turn results in more alkaline species being released. It was shown that the nanoparticulate 45S5 was able to kill $99 \%$ of the bacteria, which in this case was E.Faecalis when incubated in simulated body fluid for 100 minutes. It was also shown that the $\mathrm{pH}$ of the 45S5 nanoparticulate supernatant was 11.7 which closely coincide with calcium hydroxide which is the most commonly used disinfectant in dentistry's today. Bioactive glasses show promise as an effective antimicrobial agent that could be applied to dentistry as well as other clinical environments that require sterile equipments and settings [13].

\section{Metal Oxide Nanoparticles}

Metal oxide nanoparticles represent a new class of important materials that are increasingly being developed for 
use in research and medical applications, due to their desirable varying physical and chemical properties and also the apparent antibacterial activity. In this review only a handful of the metal oxides will be examined, but there are many more that have also been shown to exhibit antimicrobial properties [14-18].

\section{Magnesium Oxide Nanoparticles}

Nano-MgO has been involved in many different areas of research due to its novel properties, particularly in nanocomposite materials but now has been approached as antibacterial agents. $\mathrm{MgO}$ was originally known to be an antibacterial agent and it was thought that the damage caused to bacteria was due to the formation of superoxide anions on its surface; this inspired further research into understanding the mode of action that allows nano- $\mathrm{MgO}$ to exhibit antibacterial activity [14]. It was found that nano- $\mathrm{MgO}$ particles are able to take up halogen gases due to its defective natured surface and its positive charge, which results in a strong interaction with bacteria and spores as they are negatively charged. In this study the nano- $\mathrm{MgO}$ particles were produced and bromide and chloride were absorbed into the particles and then tested against E. coli, $B$. cereus and B. globigii as well as the spores from the Bacillus species. Experimental results showed that nano- $\mathrm{MgO}$ in conjunction with the uptake of halogens, in particular chloride, that effective destruction of the bacteria and bacterial spores was observed [14]. Another study was conducted which also covered the antimicrobial properties of nano- $\mathrm{MgO}$ and showed highly controlled synthesis of the particles with the ability to control particle size [15]. Through this process, using a range of Nano-Mg particle sizes ranging from $70 \mathrm{~nm}$ down to around $8 \mathrm{~nm}$, it was a observed that there was significant antimicrobial activity that actually increased the with a decrease in particle size, in particular with B.subtilis [15]. Nano- $\mathrm{MgO}$ has a great potential to be used as an antimicrobial agent, and probably more so than other metal-oxide nanoparticles showing antimicrobial activity due to the lack of required light that is necessary for other metal oxides to function, as well as the benefit of being non-toxic. It has been tested already in Phenylpropyl type interior paints and shown significant antimicrobial activity, this type of application could be applied in many medical facilities as well as households as a means to reduce contamination and pathogenesis to humans [15]

\section{Zinc Oxide Nanoparticles}

It is known that zinc oxide exhibits antibacterial activity like many other metal oxide groups and like the others only few have been scaled down to the nano size and researched further, such as $\mathrm{ZnO}$ nanoparticles. It has been shown that $\mathrm{ZnO}$ nanoparticles show significant antibacterial activity over a broad range of bacterial species and in particular against Staphylococcus aureus where it out competes five other commonly used metal oxide nanoparticles [16]. Research that was carried out by Jones [16] to test the antibacterial activity of nanoparticles as well as the impact of particle size on bactericidal efficiency. In the experiment they used four different bacteria species, which included
Staphylococcus aureus N315, Staphylococcus epidermidis 1487, Streptococcus pyogenes NZ131 and Bacillus subtilis, as well as testing four different $\mathrm{ZnO}$ particle concentrations of $0 \mathrm{mM}, 2 \mathrm{mM}, 5 \mathrm{mM}$ and $10 \mathrm{mM}$. The results showed a significant loss in viable cell numbers of all four bacteria species, with $5 \mathrm{mM}$ and $10 \mathrm{mM}$ being the most effective and $2 \mathrm{mM}$ being the worst, where it provided only a slight improvement from the control [11]. The effect of particle size was also tested using three different sized $\mathrm{ZnO}$ particles. Sizes used were $\approx 1 \mu \mathrm{m}, 50-70 \mathrm{~nm}$ and $\approx 8 \mathrm{~nm}$, and were tested on staphylococcus aureus. Results showed that the smaller particle sizes were able to reduce bacterial growth in some cases up to $99 \%$ while the larger particles for the same conditions only managed $40-50 \%$ inhibition of bacterial growth [16]. There is range of potential antibacterial applications for $\mathrm{ZnO}$ nanoparticles to be implemented into such as ointments, surface coatings, disinfectants for dentistry and in medical facilities.

\section{Silicon Dioxide Nanoparticles}

$\mathrm{SiO}_{2}$ is used very extensively in material sciences and has also been more recently used in a range of medical research such as drug delivery [17]. Nano- $\mathrm{SiO}_{2}$ is not used directly as an antimicrobial agent itself but acts as a carrier for antimicrobial and bactericidal agents due to its exceptionally porous structure. Due to this porous structure $\mathrm{SiO}_{2}$ is able to absorb ions and organic molecules quite easily, making it highly efficient and a promising carrier for antimicrobial applications. As observed in previous examples in this review, metal- containing inorganic materials has shown to be effective as antimicrobial agents; therefore a study was conducted and has implemented zinc and silver into $\mathrm{SiO}_{2}$ nanoparticles as a means of creating a high-performance bactericidal agent [18]. In the experiment conducted by a Jia [18] they synthesized two different types of $\mathrm{SiO}_{2}$ particles one that has been loaded with silver cations known as SLS and a co-absorption of zinc-silver loaded into the other, titled SLSZ. The $\mathrm{SiO}_{2}$ particles were around $20 \mathrm{~nm} \pm 5 \mathrm{~nm}$ in size and composed of $0.5 \% \mathrm{Ag}^{+}$and $4-9 \%$ of $\mathrm{Zn}^{2+}$ in the SLZS with the amount of silver ions evenly distributed in each of both SLS and SLZS. The nanoparticles were tested on two bacteria species being E.coli and Streptococcus faecalis and were incubated with two singular concentrations of $2.5 \mathrm{mg}$ and 5mg for both SLS and SLZS. The results showed that both SLS and SLZS exhibit excellent antibacterial activity, for both E. coli and S. faecalis. SLS was able to kill all the viable $E$. coli cells after a contact time of $6 \mathrm{hrs}$ when $2.5 \mathrm{mg}$ was used and was brought down to $4 \mathrm{hrs}$ when $5.0 \mathrm{mg}$ of the loaded nanoparticles were used. Likewise, the SLZS was able to decrease the contact time needed even further reducing it from $4 \mathrm{hrs}$ to $2 \mathrm{hrs}$. Results using $S$. faecalis produced were just as promising, with SLS able to kill all viable cells after an incubation period of $10 \mathrm{hrs}$ using $2.5 \mathrm{mg}$ and down to $6 \mathrm{hrs}$ using 5.0mg. Respectively for the SLZS particles the incubation time needed to kill all the viable cells was brought down from $6 \mathrm{hrs}$ using the $2.5 \mathrm{mg}$ to $2 \mathrm{hrs}$ using the amount of $5.0 \mathrm{mg}$. SLZS was noticeably the more effective of the two, which is believed to be due to an increase in antibacterial sites enhancing the contact of the particles with the bacteria so the process speed was increased. 


\section{Biological Based Antibacterial Nanomedicines}

\section{Chitosan Nanofiber}

Chitosan is a polysaccharide that is found in the exoskeleton of crustaceans and used in a range of commercial and biomedical applications such as a plant growth enhancer and even as a blood clotting agent [19]. But a recent study by Ignatova [20] has been carried out using Chitosan to create electrospun nano-fiber mats that have antibacterial activity. The experiment involves creating fibers in the range of 60$200 \mathrm{~nm}$ out of quaternized Chitosan (QCh) mixed with poly (vinyl alcohol) (PVA) and were stabilized successfully against dissolution in the aqueous environment using photomediated cross-linking. The bactericidal ability of the QCh mats is due to the damaging interaction of polycations with the negatively charged surface of bacteria which results in the loss of bacterial membrane permeability, cell leakage and eventually cell death. The effectiveness of the QCh mats was tested on S. aureus and E. coli. The mats were more effective against the gram-negative $S$. aureus, with all viable bacteria being killed after an incubation period of 60 minutes, while the gram-positive $E$. coli were destroyed after 120 minutes. The results highlight the potential of electrospun mats to be used for wound-dressings as they are able to reduce the threat of secondary infection by bacteria. Additional chitosan also provides all the desirable properties that are required for fast wound healing that is non-toxic, has hemostatic activity, biodegradability and the ability to affect macrophage function [20].

\section{Targeted Drug-Carrying Phage Medicines}

Various specific indicator cultures were tested for providing clear plaques after phage infection. Specific lytic mixture of bacteriophages was prepared using the induced, modified and laboratory variants of phages in the past [21$26]$. There are currently many highly antibacterial agents that have been developed but due to the lack of selectivity they are too potent to be used clinically. A way to overcome this shortcoming is to increase the selectivity of the agent by targeted therapy which is indeed what a group of researchers have accomplished. Yacoby [21] has devised an efficient targeted drug platform to address the requirements of targeted drug delivery, in that it is able to selectively bind to its target, provide large drug delivery payload and timely drug release. Their approach to such criteria was in the development of a drug-carrying phage nanoparticle that was successfully able to inhibit the growth of both gram-negative and gram-positive bacteria [20]. A phage was used as the drug-carrier as it has nanometric parameters that offer a unique and excellent drug-carrying ability, when compared to other particle drug-carrying devices that are commonly used such as liposomes or virus-like particles [19, 20]. This feature was also enhanced by use of clever chemistry through the introduction of amino sugar-based aminoglycosides as branched, hydrophilic linkers. This addition enabled two added benefits that are essential to the criteria required for a targeted drug carrying system, firstly providing the salvation of hydrophobic materials, which in this case is the drug chloramphenicol. This in turn enables a far greater uptake of the drug to the phage in aqueous solution, while also increasing the drug-carrying capacity of the phages from around 3000 molecules of chloramphenicol/ phage to 40,000 [20]. The targeting system uses microbe specific polyclonal antibodies that target a broad range of pathogenic bacterium. In this experiment they tested the drug-carrying phage nanoparticles against three pathogenic bacteria, which included staphylococcus aureus, streptococcus pyogenes and E.coli. Through this they were able to achieve complete growth inhibition towards all three bacterium and are comparable to using the free drug itself, resulting in an improvement factor of 20,000 compared with the free drug alone [21].

\section{Poly-L-Lactide Nanoparticles}

Nisin is an antimicrobial protein produced by Lactococcus lactis and is known as a lantinobiotic due to the fact that it contains unusual amino acids such as lanthionine [27]. Nisin has just recently been approved by the FDA for use as a food additive to provide preservation due to its broad spectrum antibacterial activity against gram-positive bacteria. One major problem when implementing nisin as a food additive is due to its weak structural integrity and certain bacteria having a minor tolerance to its effects, hefty amount of nisin is required. But it has now been combined with the use of Poly-Lactide nanoparticles in a study by Salmaso [27]. Poly-L-Lactide was used as it is biodegradable and nontoxic and could be used in certain applications of interest, as well as being easily producible. The nisin loaded poly-L-lactide nanoparticles were produced using a mixed solvent method implementing super critical $\mathrm{CO}_{2}$, and particles were shown to be of low diameter, low dispersity and were capable of loading high protein quantities. The average particle size was found to be between $250-400 \mathrm{~nm}$. They were then tested for their antibacterial properties against Lactobacillus using two nisin loaded concentrations of $20 \mathrm{mg}$ of a $5 \%$ and $20 \mathrm{mg}$ of $20 \%$ nisin loaded particle. Results of the experiment showed a significant improvement in the antibacterial activity time compared with the free nisin, with free nisin being active for around 7 days to an increased 40 days with the use of the nisin loaded in the poly-L-lactide nanoparticles. There is a considerable potential for this technique in protein formulation as shown by the nisin loaded poly-L-lactide nanoparticles, with observed results demonstrating improved protein stabilization and prolonged antibacterial activity, thus allowing such a compound to be applied in food and pharmaceutical preservation [27]. There have also been similar studies conducted using biodegradable polymers in conjunction with antibacterial agents with similar results observed. For example a study using poly Lactide-co-glycolide nanoparticles loaded with rifampicin is also an antimicrobial protein that originates from bacteria [28].

\section{DIAGNOSTICS}

Nonspecific and nonsensitive medical diagnosis problems are a quest of all civilizations. Nanoscience is one of the major areas of present scientific progress and should shortly result in essential advancement for the benefit of human health. The biomedical applications of metal nanoparticles started in the 1970s with the use of nanobioconjugates after the discovery of immunogold labeling by Faulk 
and Taylor [28]. Nanotechnology has brought a key contribution, a crucial property being the plasmon absorption and scattering of gold nanoparticles. In this review, we will concentrate our attention on gold nanoparticles, quantum dots, dye-doped fluorescent silica nanoparticles and carbon tubing.

\section{Gold Nanoparticles}

Nanodiagnostics can be defined as the use of nano-sized materials, devices or systems for diagnostics purposes. It is a burgeoning field as more and improved techniques are becoming available for clinical diagnostics with increased sensitivity at lower cost. The impact of advances in nanotechnology is particularly relevant in biodiagnostics, where nanoparticle-based assays have been developed for specific detection of bioanalytes of clinical interest. Gold nanoparticles show easily tuned physical properties, including unique optical properties, robustness, and high surface areas, making them ideal candidates for developing biomarker platforms. Modulation of these physicochemical properties can be easily achieved by adequate synthetic strategies and provides gold nanoparticles advantages over conventional detection methods currently used as $\mathrm{a}$ in the clinical diagnostics. The surface of gold nanoparticles can be tailored by ligand functionalization to selectively bind biomarkers. Thiol-linking of DNA and chemical functionalization of gold nanoparticles for specific protein/antibody binding are the most common approaches [28-30]. Gold nanoparticles used as basis for the development of methodologies suitable for application in clinical diagnosis with three different approaches; 1) utilization of the gold nanoparticles when color change upon aggregation, the best characterized example of being gold nanoparticles functionalized with ssDNA capable of specifically hybridizing to a complementary target for the detection of specific nucleic acid sequences in biological samples; 2) use of gold nanoparticles as a core/seed that can be tailored with a wide variety of surface functionalities to provide highly selective nanoprobes for diagnosis; and 3) utilization of AuNPs in electrochemical based methods that can be coupled with metal deposition for signal enhancement. The latest approaches were recently reviewed in details [30-32]. These critical reviews provide an overall survey of the basic concepts and up-to-date literature results concerning the very promising use of gold nanoparticles (AuNPs) for medicinal applications. It includes AuNP synthesis, assembly and conjugation with biological and biocompatible ligands, plasmon-based labeling and imaging, optical and electrochemical sensing, diagnostics, therapy (drug vectorization and DNA/gene delivery) for various diseases, in particular cancer (also Alzheimer, HIV, hepatitis, tuberculosis, arthritis, diabetes) and the essential in vitro and in vivo toxicity. Gold nanoparticles exploit their unique chemical and physical properties for transporting and unloading the pharmaceuticals. First, the gold core is essentially inert and non-toxic. A second advantage is their ease of synthesis; monodisperse nanoparticles can be formed with core sizes ranging from $1 \mathrm{~nm}$ to $150 \mathrm{~nm}$. Further versatility is imparted by their ready functionalization, generally through thiol linkages (vide post). Moreover, their photophysical properties could trigger drug release at remote place [30-32].

\section{Quantum Dots}

Quantum dots seem to be very promising for a range of different applications, particularly in medical diagnostics. Most of the research focus has been on the implementation of quantum dots in diagnostics of cancers and microarray technologies, but research has also been conducted on using quantum dots as the components of an effective bacterial diagnostic system. Quantum dots (QDs) are semiconducting crystals such as $\mathrm{CdSe}, \mathrm{CdTe}, \mathrm{CdS}, \mathrm{ZnSe}, \mathrm{PbS}$ and $\mathrm{PbTe}$ that typically have diameters that range between $2 \mathrm{~nm}$ and $10 \mathrm{~nm}$. QDs exhibit size-dependent optical and electronic properties which make them highly desirable for fluorometric sensor applications, as wavelength emission bands can be precisely tuned by variation in size and composition. In addition to this tunable fluorescence, they have many other advantageous properties when compared with other commonly used fluorescent labels. Their fluorescence is very bright, about 10-20 folds brighter than organic fluorophores and emission signals are significantly resistant to photo bleaching, which in turn provides the ability to use multiple color staining, while maintaining high contrast between objects, such as organs. Another fundamental feature of QDs is the possession of broad absorption spectra while maintaining specific emission spectra, which allows for the use of multiple QDs that could have differential targets and fluoresce at different wavelengths but can use the same excitation light source [33].

There has not been a great deal of research done exploring the use of QDs for bacterial diagnostics but there have been some researches that have shown promise in the area. Firstly, a study conducted by Hahn [34] was aimed at being able to detect a single bacterial pathogen with semiconductor QDs. The experiment involved the use of a CdSe core with an outer shell of $\mathrm{ZnS}$, this was then functionalized further to have a polymer coating with attached streptavidin and antibodies for targeting specific bacteria types. The bacteria targeted in this experiment were a strain of E. coli $0157: \mathrm{H} 7$, so antibodies used to target the specific bacteria were used known as anti-E coli o157:H7. Observed results were impressive, boasting an increase in the sensitivity by 2 orders of magnitude over similar organic fluorophore. Even when a greater amount of the organic fluorophores were used they weren't able to match the signal strength or match the detection limits of the functionalized QDs. Overall results showed that QDs were very stable as well as highly sensitive and being able to detect single bacterium, it should also be noted in this case that no protein function or QD emission was effected by cell labeling [35].

In another separate study conducted by Edgar [35] they created a QD that is functionalized in almost the same way as mentioned in the previous study and it was also composed of a $\mathrm{ZnS}$ shell and coated with streptavidin. Although in addition, it also incorporated biotinylated bacteriophages to produce biotin-tagged phage quantum-dot nanocomplexes (Fig. 2). Once the phage-QD complexes have bound with the bacteria it is possible to use any of microscopy, spectroscopy or flow cytometry to observe the bacteria. Due to the addition of biotin-phages with the quantum dot complexes it improves the signal amplification, while still maintaining highly specific targeting, due to the larger number of QDs on the targeting carrier. The results were impressive showing 
enhanced detection of bacterial cells, in this case $E$. coli, but through the use of alternative phages it could be possible to detect a range of different bacterium species. The technique showed an improvement in sensitivity, observed results were able to detect as little as 10 cells of $E$. coli per milliliter within approximately 1 hour [36]. The results highlight the potential of using a highly sensitive detection system that could be used in a clinical environment, such as hand-held device that would be able to quickly and effectively detect pathogenic bacteria. In another more novel way of using QDs as a bacterial diagnostic agents conducted by Dwarakanath, works on the basis of a notable change in the emission spectra of QDs when bound to a bacteria by either aptamers or antibodies [36]. In the experiments conducted to research the phenomena of change in emission wavelength, it was shown to be independent of aptamers or antibody size. Sizes of aptamers and antibodies from $900 \mathrm{kDa}$ down to as small as $18 \mathrm{kDa}$ still exhibit a shift in the emission spectra. This study highlights the potential for a specific bacterial diagnostic system based on the shifts of emission spectra from QDs [37].

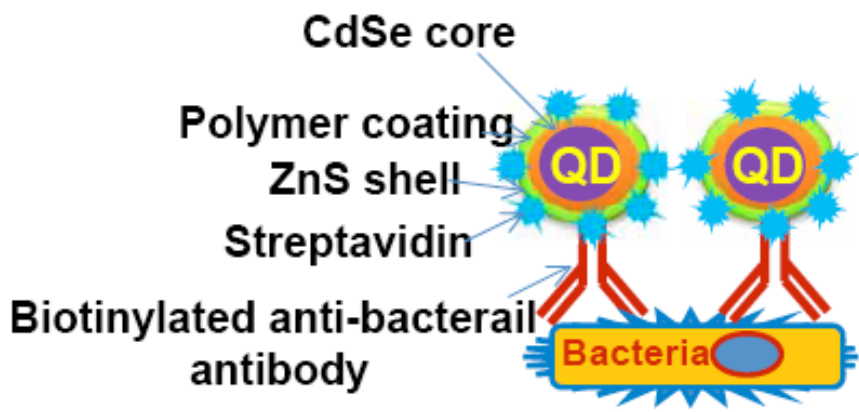

Fig. (2). Labeling of bacteria cells with biotinylated anti-bacterial antibodies and streptavidin-conjugated $\mathrm{CdSe} / \mathrm{ZnS}$ core/shell QDs.

\section{Dye-Doped Fluorescent Silica Nanoparticles}

There are currently many silica nanoparticle based bioanalysis systems and all of which are different techniques and materials to alter the mode and purpose of the particles action. As stated above, there are a range of different Silicabased nanoparticles, but for the purpose of this review it will be limited to the ones that have been researched in reference to bacterial diagnostics, just to note there is a great summary of most of the silica based nanoparticles being used in bioimaging in a review by Yan [38]. In this review it will be mainly focusing on the use of dye-doped silica nanoparticles will be mainly focussed, due to the fact that there has been a considerable amount of research conducted on them and that they have been observed to be quite effective. It should also be mentioned that although dye-doped silica nanoparticles are the same in principle but they can differ quite synthetically and in terms of results as well. Silica nanoparticles are used due their appropriate properties for the production of targeted bioimaging units. It was shown that they are also able to successfully trap dyes within the silica matrix, which in turn provides an effective barrier significantly reducing dye leakage. The reduction in dye leakage also means an improvement in the reduction of photobleaching and photodegradation, which enable their use for prolonged bioimaging and analysis work. Another feature that should be noted is the ability to functionalize the surface with relatively simple chemistry, as a range of functional groups can be added to add extra functionality by conjugation, such as a targeting ligand or a protecting molecule could be used for example. It has also been found in a study performed by Yao that the emission intensity of dye-doped silica NPs were equivalent to the use of 39 QD particles, which is due to the large packing number of dye molecules within the Silica NPs [39]. As mentioned previously, Silica nanoparticles are being used for a range of bioimaging and bioanalysis purposes although not a considerable amount was used in the detection of bacteria. In the study conducted by Yao they have created unique silica NPs which they entitled FloDots. They test the ability of bioconjugated FloDots as a means of rapid and specific determination of a single bacteria cell. In the experiment they used E.coli 0157:H7 as the target bacteria and use antibodies that target an antigen present on the surface of the bacteria. Results observed showed that it was possible to detect a single bacterium within 20 minutes and was confirmed by plate-counting method [39]. Wang [40] also showed the dye-doped silica NPs could be used as a method of monitoring pathogenic bacteria, with high sensitivity and short amount of incubation time. In their study they employed multicolored FRET (fluorescence resonance energy transfer) silica NPs, which employs the use of using a ratio of three dyes encapsulated in the silica NPs to produce unique colors upon excitation. Three types of FRET NPs were produced with different ratios of the three dyes; they were also further conjugated with PEG-streptavidin-biotinIgG complex. Corresponding antibodies from one of the three species of bacteria were used as the targeting molecule; in this case they used the bacteria E. coli, S. aureus and Salmonella typhimurium. Now that the triple-dye-doped NPs have a specific antibody correlating only to one particular bacterium as well as each particle having its own alternative ratio of the three dyes, it was possible to match a particular color emission with a specific bacteria type. The three bacterial species were incubated for $30 \mathrm{~min}$ along with the three-antibody labeled NPs and when viewed under a confocal microscope it was observed that bacteria were covered with their specifically colored NPs corresponding to their antigen present on the surface of the bacteria. The study highlights the potential of such a multiplexed diagnostic system, which is able to quickly and accurately identify a particular bacteria, such a system could be applicable for commercial use and for diagnostic purposes in regards to human health and livestock [40].

\section{Carbon Nanotubes}

As mentioned previously, new carbon nanotube applications are continuously being discovered, as the unique properties are of high interest. Carbon nanotubes have been applied as diagnostic tools in a range of areas of medicine such as cancer and other human pathogens in vitro, but not much has been done in the way of bacterial diagnostics in vivo. A study to test the effectiveness of photothermal antimicrobial nanotherapy and nanodiagnostics using selfassembling carbon nanotubes was performed by Kim [41] and has shown as an effective method of being able to achieve both bacterial diagnosis and destruction. The study involved the use of dispersed and shortened carbon nanotubes (dsCNTs) and minimally treated carbon nanotubes 
clusters that when in solution with bacteria are able to cluster at the bacterial surfaces or alternatively when using the CNT clusters which are able to form bacterial clusters. Once the bacterial or CNT clusters have formed they can be used to detect a single bacterium and or can be used to destroy the pathogenic bacteria. The process works by taking advantage of the responsiveness of carbon nanotubes to inherent near infrared lasers, which when targeted and applied causes observed bubbling phenomena that is able to completely destroy bacterial cells. Another advantage of this technique is due to the self-binding affinity of CNTs with bacteria it is unnecessary for any bioconjugation of targeting molecules, although if required for species specific purposes it is possible to do so. The study highlighted the potential application of CNT with pulse lasers as effective photothermal contrast agents that are able to self-deliver to the bacterial infection site and could be applied in the detection of a single bacterial cell in real-time [41]. Another similar study has been completed which used the same techniques of photothermal carbon nanotubes but employs targeted gold nanoparticles to do the job, and was shown to be equally effective [42].

\section{LIMITATIONS OF NANOTECHNOLOGY}

Nanomedicine, incarnation of the nanotechnology based concepts has the potential to fix innumerable problems especially in the field of medicine that remain a challenge till date, but like any other application of scientific advancement, nanotechnology has its own limitations. Possible effects of this technology could be in terms of power budget concerns of human body, where our body system utilizes certain amount of energy for its metabolic activities. The nano based application for the treatment of health aliments include development of nanomotors or nanorobots that function at cellular level which can lead to energy utilization at least 10 times the normal range, therefore creating an energy imbalance. Nanoparticles systems, comprised of multiple components which are less than $100 \mathrm{~nm}$. At this scale there is an extremely large volume to surface ratio that can manifest profound effects on the interactions of nanoscale devices with the biological counterparts. In vivo studies conducted on exposure of carbon nanotubes (CNT's) resulted in inflammation and toxic side effects in lungs and cardiovascular systems of mice, the assessment of CNT on human keratinocytes have revealed the toxic side effects invitro [43]. Cerium dioxide nanoparticles used as a promising radio -, cardio -, and neuro - protectant showed geno toxicity in terms of DNA damage towards human dermal fibroblasts by generation of reactive oxygen species leading to an oxidative stress [44]. In vitro studies with poly (butylcyanoacrylate) nanoparticles coated with polysorbate 80 cross the blood brain barrier via plasma adsorption of apolipoproteins resulting in receptor mediated endocytosis [45]. Nanoparticles are known to show increased toxicity due to their increased surface area. Nanoparticles formulations prepared from copper, cobalt, titanium oxide and silicon oxide had an inflammatory and toxic side effect on cells [46]. Engineering nanoparticles react with various components of immune system for instance, release of proinflammatory and inflammatory cytokines, inflammogenic effects of cobalt and nickel nanoparticles, stimulation of
TNF alpha secretion from macrophages by hydroxyapatite crystals that can lead to activation of phagocytes, generation of allergic mechanisms by carbon nanoparticles. Thus, nanoparticles have immunomodulatory potential that stimulate or suppress immune system which are undesirable effects in human body. Multiwalled carbon nanotubes suppress $\mathrm{T}$ cells in the immune system [47].

Titanium dioxide/zinc oxide nanoparticles used in cosmetics are known to possess oxidative DNA damaging capacity in vitro and in cultured human fibroblasts. Exposure of skin to single walled carbon nanotubes caused dermal toxicity and morphological changes due to oxidative stress. In vitro studies conducted in mice and rats caused granulomas after exposure to carbon nanotubes. Crystalline silver nanoparticle - related cytotoxicity in lesioned skin, human fibroblasts and keratinocytes [48]. Engineered nanoparticles are known to posses major genotoxic effects such as interaction with DNA and non DNA targets, their interaction with components associated with cell growth cycle such as centromeres, cytoskeleton, microtubules etc induce polyploidy and aneugenic events during cell division. Some of the other possible effects of nanoparticles include Metal release, desorption of organic components, interaction with $\mathrm{SH}$ groups and $\mathrm{Zn}$ fingers of key proteins, saturation of metallothionein, changes in DNA methylation, secondary genotoxic effects through inflammation and activation of leukocytes, HIF stabilization through hypoxia-like effects, apoptosis/ necrosis and inhibition of key receptors/enzymes [49]. Although, various above discussed limitations and unforeseen problems that can arise from nanotechnology and its applications in medicine, its derivative technologies have a promising impact to solve more problems than they will create.

\section{CONCLUSION}

Nanomedicine has provided new avenues for antibacterial therapeutics and diagnostics as well as enhancing current systems through the use of new novel nanomaterials. A variety of potential antibacterial therapeutics has been mentioned in the review from both synthetic and biologically inspired materials that have been engineered to a nanoscale. Quite a number of the therapeutics reviewed in this paper have been inspired from the bulk materials themselves such as silver and bioactive glasses, as there antibacterial activity was already recognized, but it was shown to be optimized when present in nanometric scales. High percentages of bacterial inhibition were demonstrated, and in some cases were found to completely inhibit growth of a variety of gram-negative and gram-positive bacteria species. Some of these developing techniques will find more success than others as it is only early days for all of the reviewed therapeutics, but if successful, will have a collection of uses such as ointments, sterilizing paints and materials, as well as the sterilization of medical equipments and clinical environments and have even showed a promise as potentially being applied in vivo medical therapeutics.

Also examined in the paper was the research performed in the area of potential bacterial diagnostic systems. With the research conducted through the optimization of functionalized nanoparticles impressive results were able to be 
obtained, exhibiting ultra sensitivity, rapid detection and beneficial properties for bioimaging, such as improved fluorescence. Best results showed that a single bacterium could be detected within a rapid time frame, as well as the use of multiple colored dyes to simultaneously detect three species of bacteria. A potential application for these new rapid and ultrasensitive bacterial diagnostics systems could be the ability to create a hand-held device capable of detecting a variety of bacteria species that the required can be used easily and quickly within the doctor's office, so that treatment if needed can be administered as soon as possible.

As with most new researches there are a range of hurdles to overcome and antibacterial therapeutics and diagnostic medicine and nanomedicine are not exempted from this. As the nanomedicine itself is still in its infancy, a lot of researches into the safety and properties of nanotechnologies and materials still needs to be addressed. Other implications that need to be addressed include the improvement of surface modifications to nanomaterials, uniform synthesis of particles and other materials in terms of size and function, reducing the chance of non-specific binding, and preferably non-toxic and biocompatible. Although there are a number of hurdles to overcome these efforts remain high, but hopefully through the combination of these potential new antibacterial therapeutics and diagnostics, we will be able to successfully diagnose and destroy pathogenic bacteria.

\section{REFERENCES}

[1] Chun YW, Webster TJ. The role of nanomedicine in growing tissues. Ann Biomed Eng 2009; 37(10): 2034-47.

[2] Weir E, Lawlor A, Whelan A, Regan F. The use of nanoparticles in anti-microbial materials and their characterization. Analyst 2008; 133(7): 835-45

[3] Pedroso S, Guillen IA. Microarray and nanotechnology applications of functional nanoparticles. Comb Chem High Throughput Screen 2006; 9(5): 3 89-97.

[4] Kanwar J R, Mahidhara G, Kanwar R K. Recent advances in nanoneurology for drug delivery to the brain. Curr Nanosci 2009; 5(4): 441-8.

[5] Sara B, Kanwar RK, Khoshmanesh K, et al. Promises of nanotechnology for drug delivery to brain in neurodegenerative diseases Currt Nanosci 2009; 5(1): 26-32.

[6] Yacoby I, Benhar I. Antibacterial nanomedicine. Nanomed 2008; 3(3): 329-41.

[7] Seoktae K. Single-walled carbon nanotubes exhibit strong antimicrobial activity. Langmuir 2007; 23(17): 8670-3.

[8] Chipot C, Tarek M. Interaction of a peptide nanotube with a watermembrane interface. Phys Biol 2006; 3(1): p S20-5.

[9] Lyon DY, Fortner JD, Sayes CM, Colvin VL, Hughe JB. Bacterial cell association and antimicrobial activity of a C60 water suspension. Environ Toxicol Chem 2005; 24(11): 2757-62.

[10] Moghimi SM. Nanomedicine: prospective diagnostic and therapeutic potentials. Asia Pacific Biotech News 2005; 9(20): $1072-7$.

[11] Sondi I, Salopek-Sondi B. Silver nanoparticles as antimicrobial agent: a case study on $E$ coli as a model for Gram-negative bacteria. J Coll Inter Sci 2004; 275(1): 177-82.

[12] Shahverdi AR. Synthesis and effect of silver nanoparticles on the antibacterial activity of different antibiotics against Staphylococcus aureus and Escherichia coli Nanomedicine. Nanotech Biol Med 2007; 3(2): 168-71.

[13] Waltimo T. Antimicrobial effect of nanometric bioactive glass 45S5. J Dent Res 2007; 86(8): 754.

[14] Stoimenov PK. Metal oxide nanoparticles as bactericidal agents. Langmuir 2002; 18(17): 6679-86.

[15] Huang L. Controllable preparation of Nano- $\mathrm{MgO}$ and investigation of its bactericidal properties. J Inorg Biochem 2005; 99(5): 986-93.
[16] Jones N. Antibacterial activity of $\mathrm{ZnO}$ nanoparticle suspensions on a broad spectrum of microorganisms FEMS. Microbiol Lett 2008; 279(1): 71-6.

[17] Anglin EJ. Porous silicon in drug delivery devices and materials. Adv Drug Deliv Rev 2008; 60(11): p 1266-77.

[18] Jia $\mathrm{H}$. The structures and antibacterial properties of nano- $\mathrm{SiO}_{2}$ supported silver/zinc silver materials. Dent Mater 2008; 24(2): 244-9.

[19] Prabaharan M. Review Paper: Chitosan derivatives as promising materials for controlled. Drug Deliv J Biomat Applicat 2008; 23(1): 5-36.

[20] Ignatova M. Electrospun nano-fibre mats with antibacterial properties from quaternised chitosan and poly(vinyl alcohol). Carbohydr Res 2006; 341(12): 2098-107.

[21] Yacoby I. Targeted Drug-Carrying bacteriophages as antibacterial nanomedicines. Antimic Agents Chemother 2007; 51(6): 2156-63.

[22] Bar H, Yacoby I, Benhar I. Killing cancer cells by targeted drugcarrying phage nanomedicines. BMC Biotechnol 2008; 8: 37.

[23] Fischetti VA. Bacteriophage lytic enzymes: novel anti-infectives. Trends Microbiol 2005; 13(10): 491-6.

[24] Dixon B. New dawn for phage therapy. Lancet Infect Dis 2004; (3): 186.

[25] Merril CR, Scholl D, Adhya SL. The prospect for bacteriophage therapy in Western medicine. Nat Rev Drug Discov 2003; 2(6): 489-97.

[26] Rippon JE. A new serological division of Staphylococcus aureus bacteriophages: group G. Nature 1952; 16170(4320): 287.

[27] Salmaso S. Nisin-loaded poly-l-lactide nano-particles produced by $\mathrm{CO}<\mathrm{sub}>2</ \mathrm{sub}>$ anti-solvent precipitation for sustained antimicrobial activity. Int J Pharma 2004; 287(1/2): 163-73.

[28] Faulk WP, Taylor GM. An immunocolloid method for the electron microscope. Immunochemistry 1971; 8(11): 1081-3.

[29] Baptista P, Pereira E, Eaton P, et al. Gold nanoparticles for the development of clinical diagnosis methods. Anal Bioanal Chem 2008; 391(3): 943-50.

[30] Ghosh P, Han G, De M, Kim CK, Rotello VM. Gold nanoparticles in delivery applications Adv Drug Deliv Rev 2008; 60(11): 130715.

[31] Laurent S, Forge D, Port M, et al. Magnetic iron oxide nanoparticles: synthesis, stabilization, vectorization, physicochemical characterizations, and biological applications. Chem Rev 2008; 108(6): 2064-110.

[32] Boisselier E, Astruc D. Gold nanoparticles in nanomedicine: preparations, imaging, diagnostics, therapies and toxicity. Chem Soc Rev 2009; 38(6): 1759-82.

[33] Esmaeili F, Hosseini-Nasr M, Rad-Malekshahi M, Samadi N, Atyabi F, Dinarvand R. Preparation and antibacterial activity evaluation of rifampicin-loaded poly lactide-co-glycolide nanoparticles. Nanomedicine 2007; 3(2): 161-7.

[34] Hahn MA, Tabb JS, Krauss TD. Detection of single bacterial pathogens with semiconductor quantum dots. Anal Chem 2005; 77(15): 4861-9.

[35] Edgar R, MsKinstry M, Hwang J, et al. High-sensitivity bacterial detection using biotin-tagged phage and quantum-dot nanocomplexes. Proc Natl Acad Sci USA 2006; 103(13): 4841-5.

[36] Dwarakanath S. Quantum dot-antibody and aptamer conjugates shift fluorescence upon binding bacteria. Biochem Biophys Res Commun 2004; 325(3): 739-43

[37] Tallury P, Payton K, Santra S. Silica-based multimodal/ multifunctional nanoparticles for bioimaging and biosensing applications. Nanomed 2008; 3(4): 579-92.

[38] Yan J, Estévez MC, Smith JE, et al. Dye-doped nanoparticles for bioanalysis. Nano Today 2007; 2(3): 44-50.

[39] Gang Y. FloDots: luminescent nanoparticles. Anal Bioanal Chem 2006; 385(3): 518-24

[40] Wang L. Fluorescent nanoparticles for multiplexed bacteria monitoring. Bioconj Chem 2007; 18(2): 297-301.

[41] Kim JW, Shashkov EV, Galanzha EI, Kotagiri N, Zharov VP. Photothermal antimicrobial nanotherapy and nanodiagnostics with self-assembling carbon nanotube clusters. Lasers Surg Med 2007; 39 (7): 62-634.

[42] Zharov VP. Photothermal nanotherapeutics and nanodiagnostics for selective killing of bacteria targeted with gold nanoparticles. Biophys J 2006; 90(2): 619-62.

[43] Bindu MN, Muhammed STK, Wojciech GL, et al. Toxicity evaluation of gold - dendrimer composite nanodevices invitro - 
difference found between tumour and proliferating endothelial cells. Nanotoxicology 2009; 3(2): 139-51.

[44] Melanie A, Jerome R, Thierry O, et al. $\mathrm{CeO} 2$ Nanoparticles Induce DNA damage towards human dermal fibroblasts in vitro. Nanotoxicology 2009; 3(2): 161-71.

[45] Silva GA. Nanotechnology approaches for drug and small molecule delivery across the blood brain barrier. Surg Neurol 2007; 67: 113-6.

[46] Ochekpe NA, Olorunfemi PO, Ngwuluka NC. Nanotechnology and drug delivery part 1: background and applications. Trop J Pharma Res 2009; 8(3): 265-74.
[47] Dwivedi PD, Misra A, Shanker R, Das M. Are nanomaterials a threat to the immune system? Nanotoxicology 2009; 3(1): 19-26.

[48] Bharali D, Khalil M, Gurbuz M, Simone TM, Mousa SA. Nanoparticles and cancer therapy: a concise review with emphasis on dendrimers. Int J Nanomed 2009; 4: 1-7.

[49] Gonzalez L, Lison D, Kirsch-Volders M. Genotoxicity of engineered nanomaterials: a critical review. Nanotoxicology 2008; 2(4): 252-73.

Received: August 26, 2009

(C) Matthews et al.; Licensee Bentham Open.

This is an open access article licensed under the terms of the Creative Commons Attribution Non-Commercial License (http://creativecommons.org/ licenses/by-nc/3.0/), which permits unrestricted, non-commercial use, distribution and reproduction in any medium, provided the work is properly cited. 